\title{
INTEGRAL CLINICAL INVESTIGATION
}




\title{
INTEGRAL CLINICAL INVESTIGATION
}

\section{An Aspect of Pananthropic Medicine}

\author{
John G. Howells \\ Director, The Institute of Family Psychiatry, Ipswich
}


(C) John G. Howells 1982

Softcover reprint of the hardcover 1st edition 1982 978-0-333-29446-8

All rights reserved. No part of this publication may be reproduced or transmitted, in any form or by any means, without permission.

First published in 1982 by

THE MACMILLAN PRESS LTD

London and Basingstoke

Companies and representatives

throughout the world

Typeset in Baskerville by

Vantage Photosetting Co. Ltd.

Southampton and London

ISBN 978-0-333-29447-5

ISBN 978-1-349-16817-0 (eBook)

DOI $10.1007 / 978-1-349-16817-0$

The paperback edition of the book is sold subject to the condition that it shall not, by way of trade or otherwise, be lent, resold, hired out, or otherwise circulated without the publisher's prior consent in any form of binding or cover other than that in which it is published and without a similar condition including this condition being imposed on the subsequent purchaser. 


\section{Contents}

Foreword

Acknowledgements

Introduction

SECTION I: BASIC CONSIDERATIONS

PART ONE Some General Principles of Pananthropic Medicine 3

Chapter 1. Psychic and Somatic Components in Disease 4

Predominantly Somatic States (I) 5

Predominantly Psychic States (II) 6

Mixed States of I and II $\quad 6$

Additional General Comment $\quad 8$

Conclusions $\quad 8$

Chapter 2. The Morbid Process-One Process (Psychic and Somatic) 11

The Agent 13

The Fabric 13

The Indicators $\quad 15$

The Diagnosis 16

Chapter 3. Nosology in Psychic Medicine 17

Neurosis $\quad 17$

Psychosis $\quad 18$

Cryptogenic States $\quad 19$

Conclusion $\quad 21$

Chapter 4. The Meeting of Patient and Doctor 22

$\begin{array}{ll}\text { PART TWO The Clinical Interview } & 27\end{array}$

Chapter 5. The Clinical Interview 28

Introduction $\quad 28$

Functions of Clinical Interviews $\quad 28$

Types of Interviews in Clinical Investigation $\quad 29$

The Interviewer and His Role 36

Organisation $\quad 40$

Learning the Art of Interviewing 49 
vi Contents

SECTION II: THE INTEGRAL CLINICAL INVESTIGATION

PART ONE The Individual

Summary of the Integral Clinical Investigation of the Individual Patient in Ten Steps

Introduction

Step I. General Data 57

Step II. The Illness as Seen by the Patient 59

Step IIA. The Presenting Complaint or

Complaints 60

Elements in the Description of Symptoms 62

Elements in the Description of Pain 64

Elements in the Description of a Swelling, Lump or Mass

64

Elements in the Description of Fluids 65

Step IIB. The Additional Complaints 66

Step IIC. Systematic Enquiry for

Symptomatology

Schedule for Systematic Elucidation of Individual Symptomatology $\quad 68$

Psychic Dysfunction $\quad 69$

Somatic Dysfunction $\quad 81$

Step IID. Historical Course of the Illness 97

Step III. The Patient as Fabric of His Illness 100

Step IIIA. The Patient 101

Step IIIB. The Life Experience of the Patient 103

Step IV. Preliminary Diagnosis 106

Step V. Examination 107

One Plan for Geographical Examination of a Patient

Schedule for the Examination $\quad 110$

Psychic Dysfunction 111

Somatic Dysfunction $\quad 124$

Step VI. Substantive Diagnosis $\quad 155$

Step VII. Special Investigations 162

Step VIII. Extended Diagnosis 166

Step IX. Formal Recording of the Case Material 167

The Systematic Clinical Record (SCR) 168

The Problem Orientated Medical Record

(POMR)

172

Confidentiality $\quad 174$

Conventional Symbols $\quad 175$

Diagrams $\quad 180$

Step X. Informing the Patient 184

Addendum to Part One 185

I. Investigation in Special Circumstances $\quad 185$

II. History Taking from Relatives and Others 187 
III. Use of the Investigation with Patients in Primitive Cultures

Summary of the Integral Clinical Investigation of the Individual Patient in Ten Steps

Summary of the Integral Clinical Investigation of the Family in Ten Steps

Introduction

Step I. General Data

Step II. The Illness as Seen by the Family 201

Step IIA. The Presenting Complaint or

Complaints

Step IIB. The Additional Complaints 202

Step IIC. Systematic Enquiry for Symptomatology

Schedule for Systematic Elucidation of Family Symptomatology

Step IID. Historical Course of the Family Illness 207

Step III. The Family as Fabric of Its Illness 210

Step IIIA. The Family 211

Schedule 211

Step IIIB. The Life Experience of the Family 213

Schedule $\quad 214$

Step IV. Preliminary Diagnosis 218

Step V. Examination $\quad 219$

Schedule for the Examination $\quad 220$

Step VI. Substantive Diagnosis 222

1. Identification of Pathological Data 223

2. The Syndrome 224

3. Understanding the Disorder 225

4. Description and Labelling of the Disorder or Disorders

5. Management 227

6. Prognosis 228

Step VII. Special Investigations 230

Step VIII. Extended Diagnosis 233

Step IX. Formal Recording of the Family Case Material 234

Step X. Informing the Family 236

Summary of the Integral Clinical Investigation of the Family in Ten Steps

$\begin{array}{ll}\text { PART THREE The Morbid Process } & 239\end{array}$

The Morbid Process $\quad 240$

Intention $\quad 240$

Psychopathology 241 
viii Contents

Instructions $\quad 245$

1. Method 245

2. Approaches 246

3. Technique 247

4. Time 249

5. Number of Interviews 249

6. Diagnosis and not Therapy 250

References

251

Author Index

255

Subject Index

256 


\section{Foreword}

The knowledge that psychological factors, including personality, may play a role in the development of disease dates back to ancient Graeco-Roman medicine. In modern times the spectacular developments of knowledge in pathology, bacteriology and the biochemical basis of illness have dominated medical thought regarding the causation and treatment of disease. An increasing volume of research has attested to the importance of emotional factors in the development of various physical diseases and has also paid attention to the emotional impact on the person of physical disease itself, and it has become clear that in many illnesses both psychosomatic as well as somatopsychic effects are important.

From many directions it is frequently pointed out that the medical man is not sufficiently aware in his diagnosis or treatment of the emotional factors in physical illness and of the emotional illness itself. The doctor is exhorted to have more understanding, for example, of the reasons for delay in seeking medical attention, of the importance of emotional factors in the aetiology of physical illness and the reduction of emotional stress in the management of pain, or in bereavement, or in terminal illness, or in accident proneness, or in the handling of mastectomy and hysterectomy patients, or in the care of handicap or disfigurement, and so forth.

If the doctor is to be effective in taking account of psychological and social effects in medicine and surgery and in emotional illness, then their assessment must be an integral part of the diagnostic process. Every doctor on every occasion should always assess emotional factors with somatic factors-thus employing a holistic diagnostic approach, the appropriate approach for the complete doctor.

The need to take into account psychological, social and physical aspects of the illness is the basis of the Integral Clinical Investigation (ICI) fully described in this book. It follows that those components of the illness which are equal parts of the whole must be assessed in total investigation and improved in total treatment.

The Integral Clinical Investigation (ICI) has three parts. Part One recognises that it is the individual who in most cases seeks help from his medical attendant. It allows for total assessment, body and psyche, of all age groups. The final diagnosis takes account of organic pathology and psychopathology and influences the doctor to plan treatment on an holistic basis. Part Two acknowledges the increasing importance of the family. The Black report, entitled Inequalities in Health (DHSS, 1980) noted the evidence that respiratory disease is commonly to be found in all or most members of affected families, and asked 'How common is this familial aggregation of ill health?'. Litman (1974) has noted that the 'family constitutes the most important social context 
within which illness occurs and is resolved'. This illness can be somatic or emotional. Part Two is a family diagnosis embracing both the somatic and psychic components. Part Three helps the doctor in his final step in diagnosis. Having established the extent of the organic pathology and the psychopathology he has to ask what is the nature of this process. Somatic pathology would be known to him and Part Three supplies the ready means of obtaining the psychopathology which is unique for each individual or family. Parts One and Two have ten clear matching steps. The schedules of the three Parts are preceded by a discussion of the important matters of communication and relationship.

Dr Howells, throughout his career, has taught the importance of a holistic approach to all illnesses and he has gained a world-wide reputation for his work in Family Psychiatry, as well as for his contributions on the psycho-social aspects of illness.

This book can be warmly recommended for undergraduate and postgraduate students and their teachers, and indeed for all doctors, whatever approach to medicine or surgery they practice.

Professor W. Linford Rees

\section{References}

DHSS (1980). Inequalities of Health, Report of a research working group Litman, T.J. (1974). Soc. Sci. Med., 8, 495 


\section{Acknowledgements}

My thanks are due to a number of colleagues who gave me invaluable advice on the planning and content of the manuscript. These are two psychiatric colleagues-Dr Judith Cuthill and Dr J.K. Merritt; a physician-Dr John Day; a surgeon-Mr E Leighton-John; together with two primary physicians - Dr M.L.B. Pritchard and Dr David Stuart. In the presentation of the material I was fortunate in having the experienced help of Mrs Livia Osborn. Mrs Lesley Horrocks earns my appreciation for her careful preparation of the manuscript. I acknowledge the opportunity to present the case example in Part Two of Section II by Dr Clyne and Update. It is also pleasant to record that I benefited from the constant companionship of the Butterworths Medical Dictionary. 


\section{Introduction}

A medical student's first patients make an indelible impression on the mind. I can recall mine with ease. The ulcer patient, for instance, whose frequent haematemeses had reduced him to imminent death and the lowest haemoglobin index in the experience of the pathologist. Hovering about the bedside was the anxious wife who never wished to talk to me-or her husband for that matter. The patient refused surgery. I remember the long slow struggle to get him well. He left with no word of appreciation to nurse or doctor or to me. His actions seemed inexplicable at the time. But how much I had missed! I can understand that now. Why did I not ask 'Why does he want to die?' But then my history taking had no place for questions about his feelings. I found his wife difficult. Did he? Was this the cause of his ulcer? Again my history taking took no account of feelings in the family. Essentially, this book aims to produce a complete form of investigation that includes the hitherto unexplored psychic parts of an investigation. Thus it encompasses the usual general investigation and includes in addition an assessment of the psychic component of the individual, one integrated investigation. Similarly, a full integrated appraisal of his family is possible when required.

Until the 17th century, medicine, within the limits of its knowledge, based its practice on a broad approach that embraced both the physical and psychic aspects of the patient. Thus, in an effort extending over many centuries, from the earliest recorded medical history there was an attempt to practice pananthropic medicine-medicine of the whole man. After the 17 th century, the upsurge of knowledge in the organic field diverted medicine to an almost total involvement with physical phenomena; psychic phenomena were largely overlooked. The dark age of psychiatry that ensued is slowly passing as we move back to a position lost some three centuries ago.

The lack of a holistic approach has lead not only to somatists ignoring psychic phenomena, but also, equally unfortunate, to psychiatrists ignoring somatic phenomena. In recent times there have been advocates against the practice of somatists ignoring psychic phenomena. To mention but a few, Balint, both in his writings (1968) and teaching groups for primary physicians, Browne and Freeling (1967), commenting on the doctor-patient relationship, and Shepherd and his colleagues (1959) in their epidemiological studies. A study by Maguire (1976) reveals that doctors inquired directly about the possibility of mastectomy patients being emotionally disturbed in only 5 per cent of those cases where patients had given evidence that they were distressed. Fletcher (1980) points to the particularly damaging consequences of poor communication technique on elucidating the psychological causes of disease. With regard to the second practice, psychiatrists ignoring somatic states, Maguire and Granville-Grossman (1968) have this to say: 'in a study of 
200 consecutive admissions to a psychiatric unit, the physical illness evident in $33 \%$ had only previously been diagnosed in half of these cases'. Further evidence is given by Wilkinson (1979).

The above would argue for a holistic approach. Maguire and Rutter (1976) in this context state "these findings strongly suggested that doctors ought to be trained...to educate their patients to realise that they were genuinely concerned with practical, social and psychological problems as well as physical illness'. This is the stance taken by a group of authors led by Maxwell (1976). The Cartesian paradigm of illness which separates body and mind has to go.

Pananthropic medicine goes much further than is sometimes supposed. It is not just a matter of taking account of a patient's feelings about his physical illness, or the effect of a physical illness on his psychic wellbeing, or the influence of psychic events in causing physical symptoms, or how to handle patients, or handling special situations such as death or of humanising medicine. It is much more. Its central principle is that the whole, body and psyche, can become ill. Thus the psyche can become ill. It follows that the psychic component, as a part of the whole, must be assessed in total investigation and improved in total treatment.

The desirable reorientation of medicine is held back at the first clinical step- that of investigation-as clinicians do not employ a system of investigation that embraces both psychic and somatic phenomena. The integral approach is missing in investigation and its absence sets a serious limitation on our efforts; and indeed of itself hides the paucity of our findings. Maguire and Rutter (1976) comment 'It seemed likely that most doctors tended to assume that clinical problems would fall into either a physical or psychological domain and would only rarely utilise an interviewing strategy that allowed for the possibility of both types of problem being present'. Indeed it is possible to have two forms of history taking, one employed by all departments, except the psychiatric, concentrating on the organic, and the other taught in the department of psychiatry concentrating on the psychic alone. Again the latter may heavily concentrate on a 'mental' examination, i.e. symptomatology arising from an organic cerebral disorder rather than upon a psychic, emotional, disorder.

In systems concentrating on the somatic, the psychic examination is usually left out, or done as a separate part, and then only when there are special indications. Psychic evaluation is not regarded as something to be done always. Equally, in psychiatric examination, somatic exploration is left out or included as a separate part and to be undertaken only when there are special indications. It is not regarded as something to be done always. Here we see the fragmentation of medicine.

The physician's interaction with his patient begins with investigation. Some modern texts of investigation in somatic medicine have given significant attention to the psychic without achieving complete integration, e.g. the excellent texts by Macleod (1979) and Hutchison (1980) in the United Kingdom have a chapter on the psychological state, and Morgan and Engel (1969) in the USA have emphasised the importance of good psychological interview technique. Maguire and Rutter (1976) put forward a brief model for history taking which include some material on the psychic state. 
In this book the author aims to reorientate investigation much further. The essential principle of integration is grasped and applied throughout in a new complete new system of investigation. The model includes the psyche and the soma throughout, with equal emphasis. From the evaluation of the psychic as well as the somatic presenting complaints it moves step-by-step exploring psychic and somatic elements together to the final diagnosis which is expressed in integral terms. The integral model is assumed to be a standard format to be used always by all clinicians in any field; the model however can be adjusted to go into the psychic or somatic states predominantly. The model covers not only the psychic and somatic aspects of the patient, but also his social background, indeed it differentiates the illness from the background. A similar model allows the family when necessary to be taken as the patient and explores it in an integral way. Finally, a model is put forward for the exploration of psychopathology; its use is advocated when it has not clearly emerged in the general investigation of individual or family.

The first part of the investigation described in this volume creates a framework for a fully integrated clinical examination of the individual. Careful appraisal reveals that it is meaningless to ask 'Is the patient's problem somatic or psychic?' Truly, we must ask 'What are the somatic and psychic components of the patient's disorder?'; it is rare for both not to exist together. The first aim, then, of the integral investigation is to establish the diagnosis of the individual's state of dysfunction, psychic and somatic, i.e. to say in what way the patient is not well.

It is customary to regard the individual as the optimum unit in clinical practice. There are, however, reasons for sometimes accepting the family, rather than the individual, as the basic unit. The family is an organism as well defined as an individual. A sick individual all too often represents a sick family; indeed, to treat only one sick individual in a family often merely succeeds in replacing him with another sick member of the same family. The family is a unit small enough to be studied by clinical methods; clinicians are increasingly examining it, both in its somatic and psychic components. Thus, the second part of the integral examination establishes the diagnosis of the family's state of dysfunction, psychic and somatic, i.e. in what way the family is unwell.

The physician, once a diagnosis is established, need not explore the somatic pathological process, as this can usually be inferred from already published texts. In the psychic field, however, the clinician is in unchartered territory. Therefore, the third part of the integral examination guides the clinician, when it is required, in exploring the psychic morbid process, i.e. why the psychic part of the patient, individual or family, has become unwell.

Concepts of the morbid process are not as well developed in psychiatry as they are in organic medicine. The field is complex and therefore a rich area for the speculator and the mystic. However enough knowledge is available to eschew the fanciful and to base concepts on the definable and the testable. Here the system of examination is based upon the concepts of experiential psychopathology (Howells, 1975); this regards the morbid process as having arisen from real harmful experience, all the noxious events the organism, family and individual have been subject to in life. Symbolism is discarded. The tail of a dog is not taken always to represent a phallus-it usually represents a part of a dog. Rarely a dog's tail may have been employed as a phallus in the patient's experience; even then its recall is not a symbol of the event, but 
portrays a real event. The facts, in real terms, are all. Revelation, clarification, description are permissible; to interpret, and thus change reality, in the light of dogma is forbidden.

An integral investigation raises the question of a common pathology for psychic and somatic phenomena, rather than distinct psychic and somatic pathologies. The author regards the former view as plausible. However, it would be a digression to explore this question here. Whatever view is held by the clinician the model prescribed here holds good. Should the first view be established the model would still be valid, but probably gain in precision.

Some break-off point must be established in any system of clinical investigation. Here the point taken is the passing over from general, or initial investigation, to the detailed exploration of a special area. Here we have an outline. Many excellent texts are available on the investigation of special areas. Furthermore, a book could be devoted to each of the symptoms and signs; no such detail can be offered here but is available in larger texts on investigation or in books concerned with one symptom. Again, special investigations cannot be considered in detail; many of these need a volume to themselves for a full description.

It is sometimes suggested that a record of the clinical investigation, especially if more structured, should be employed to cover other activities such as research, medical intelligence, manpower planning, medical audit, etc. This is an unwarranted intrusion. Extra functions should not be imposed on the physician and his record should not be cluttered with extraneous items and his efforts hampered by irrelevant material. These other activities should be undertaken by different personnel by different procedures. The clinical record serves clinical investigation and is a tool devised for this purpose. Furthermore an open record may destroy the basis of a satisfactory investigation, the trust of the patient.

The system of integral examination is suitable for patients of all age groups; where it is necessary to adjust the plan for a subject of a particular age this is indicated in the text. Furthermore, it can be employed with any presenting somatic or psychic symptom and the format is appropriate for all types and sizes of families. It can be translated as it stands for use in any developed country or culture; for primitive communities it is easily adjustable into simple suitable language. Finally, the system of integral examination can be used by all medical practitioners-generalist, internist, surgeon, psychiatrist and medical student. Each of them, after the general examination outlined here, can move to a more detailed exploration of his special field.

Medical schools, as the result of long experience in teaching students and continuing staff discussion, have usually evolved systems for student teaching. These special features of this integral outline-equal emphasis to psychic and somatic components, a system for family investigation, a model for illiciting psychopathology, can be grafted on to those existing well tried systems. This outline complements bedside teaching but is no substitute for it. From the clinical teaching situation arises indefinable extra qualities-priorities and essentials present in a direct way, the situation often dictates the way in which the outline should be used, flexibility in the use of the outline becomes second nature as each patient is different from the next, and abbreviation is a response to a real situation but based on a thorough understanding of the outline. 
It may be useful to list some of the special features of the integral clinical investigation:

1. The investigation is part of the approach of pananthropic medicine which in diagnosis, description of the morbid process, treatment and prognosis takes account of the total patient, somatic and psychic-a total assessment. Thus the psychic component of the disorder is given an equal place in investigation with the somatic component.

2. One format only is used for the assessment of both the psychic and somatic components of a disorder.

3. The medical model with its system for understanding illness is found to be completely satisfactory, indeed essential, in the consideration of psychic as well as somatic medicine; properly understood and employed the medical model has always encompassed both.

4. The outline encompasses multiple aetiologies of a disorder, and multiple disorders in one patient.

5. The nosology of psychic disorders takes on a form that fits more rationally into a total medical nosology. Clear distinction is made between psychic disorder proper, and insanity-a disorder probably belonging to somatic medicine.

6. Outmoded psychopathologies are replaced by the experiential psychopathology model that allows the assimilation of real events and new findings from systematic experimentation.

7. The integral clinical investigation in ten matching clear steps can be employed with families or with individuals. There is a systematic account of the individual, his family, and his social surroundings.

8. The diagnosis takes account of the normal and abnormal, health and pathology, assets and weaknesses.

9. The results of the integral clinical investigation may demand an exploration of the morbid process and, as this has to be worked out afresh in its psychic component, a framework for elucidation of this component is supplied.

10. Emphasis is given throughout to the importance of communication between doctor and patient, individual or family.

11. The investigation is suitable for index patients of all age groups.

12. It can be employed in any country or culture.

The system requires no more time for its performance than traditional systems. Furthermore, once the schema outlined here is learnt, it can be greatly abbreviated to meet the dictates of many variants of clinical practice. Its essence can be conducted in a few minutes. However, the existence of the framework adds much, by its order and coverage, even to a brief investigation of 5 minutes' duration.

The book starts by considering some general principles of investigation. A discussion of the clinical interview follows. Part One of the investigation consists of 10 steps for the clinical examination of the individual. Part Two of the investigation consists of 10 steps for the clinical examination of the family. Part Three of the investigation is an outline of a procedure for the exploration of psychopathology. 